\title{
AN EFFICIENT CONTACT MODEL FOR ROTATING MECHANISM ANALYSIS AND DESIGN IN BRIDGE CONSTRUCTION
}

\author{
SHIPING HUANG ${ }^{1,2^{*}}$, YONG TANG ${ }^{1}$, \\ ZHAOXUN YUAN ${ }^{1}$, XIAOPENG CAI ${ }^{1}$ \\ ${ }^{1}$ School of Civil Engineering and Transportation, \\ South China University of Technology, Guangzhou, China \\ ${ }^{2}$ Tsinghua University, State Key Laboratory of Tribology, \\ Beijing, China
}

Received 28 January 2020; accepted 12 May 2020

\begin{abstract}
The rotation superstructure construction method is a widespread technique in bridge engineering. The critical issue for the successful application of this technique is the contact interface analysis and design for the rotating mechanism. A semi-analytical method predicated upon obtaining a uniform distribution of pressure on the slide plates within the interface is proposed. The surface design typically generates a nonlinear stress distribution. It leads to local damage and local asperity interlocking, which increase the contact friction dramatically during the rotation. In contrast, the proposed approach provides a surface that avoids stress concentrations and is expected to reduce the material cost of the slide plates. The proposed method is verified by the Finite Element Model. It can be used in a broad area involving contacting surface design, especially in the rotating mechanism design for bridge construction.
\end{abstract}

Keywords: bridge construction, contact behaviour, friction, optimal design, rotating mechanism, slide plate.

\footnotetext{
* Corresponding author. E-mail: ctasihuang@scut.edu.cn
}

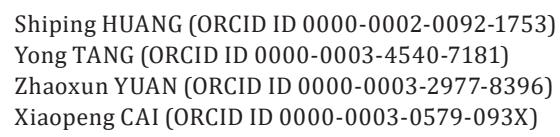




\section{Introduction}

The rotation superstructure construction method is attractive for installing bridges in many cases (Zhang \& El-Diraby, 2006). In this method, the bridge superstructure is first built in an open area parallel to the spanned obstacle (a river, a canyon, a highway, or a railway). The superstructure is then rotated into the desired position. The method promotes bridge construction in reducing impacts on traffic, safety, and overall project budget and duration. The critical challenge for the success of this method is the rotating mechanism, which involves the contact and friction problem in large contacting bodies under high pressures.

The contact mechanics of interfaces formed by joining surfaces is a fundamental problem in engineering. The pioneering work related to the contact mechanics of deformable bodies has been done by Hertz (1881, 1896), who developed the stress-displacement relationship of contact between two perfectly smooth convex elastic bodies under normal loading. Since the 1950s, numerous researches on the contact mechanism have addressed the influences of surface roughness, friction, and adhesion. Mindlin (1953) studied the contact behaviour of two smooth spheres under combined normal and shear loading. Greenwood \& Williamson (1966) introduced the statistical approach to describe the roughness of the interface, assuming each asperity had the same radius but different heights. Nayak (1971, 1973a, 1973b) utilised the random process theory (Longuet-Higgins, 1957a, 1957b) in the analysis of Gaussian roughness, which was subsequently used by Bush et al. (1975) in rough surface contact. These surface roughness analyses have also been extended to consider the effect of asperity interlocking on friction (Huang \& Misra, 2013; Misra \& Huang, 2011). At the same time, many researchers have contributed to improving the efficiency and accuracy of the computational method for the contact problem. The computational methods based on classical continuum assumptions are Finite Element Method (FEM) (Hyun et al. 2004; Wriggers \& Zavarise, 2004) and boundary element method (Banerjee \& Butterfield, 1981; Brebbia \& Walker, 2016). Molecular dynamics have been used to simulate the surface interactions, aiming to get molecular origins of the contact/ friction mechanism (Berman et al., 2015; Huang, 2017; Luan \& Robbins, 2005; Mo et al., 2009; Pastewka et al., 2012).

The motivation of research on contact behaviour is to control friction. However, strategies to control friction in practice remain a challenge (Barber \& Ciavarella, 2000; Urbakh et al., 2004). In this paper, the authors investigate the contact behaviour of the rotating mechanism to provide knowledge for efficient and optimal rotating 
mechanism design. The ideal contact surface gives a uniform stress distribution and has minimal friction. In the following sections, the authors combine the classic contact theory and computational technique with improving the contact surface design in a large contact system. The proposed method is verified by the existing computational method.

\section{Methods and materials}

\subsection{The background of the contact problem: description of the construction method}

During the bridge construction process, access to the bridge crossing area is often restricted. An example is shown in Figure 1, where a highway bridge is required to span an in-service high-speed railway line. In these cases, the rotation superstructure construction method is one of the best choices for construction. A general procedure for the bridge construction based on rotation superstructure construction method consists of follows steps:

1. foundation construction,

2. installation of the rotation mechanism,

3. pier construction,

4. supper structure construction (in this case using hanging basket construction method), and

5. rotating the structure to the desired position.

Except for Step 2 and Step 5, the other steps are conventional construction procedures.

A general rotating mechanism configuration is shown in Figure 2. The mechanism consists of a spherical hinge and the traction strand. The contact occurs between the upper spherical hinge-component and

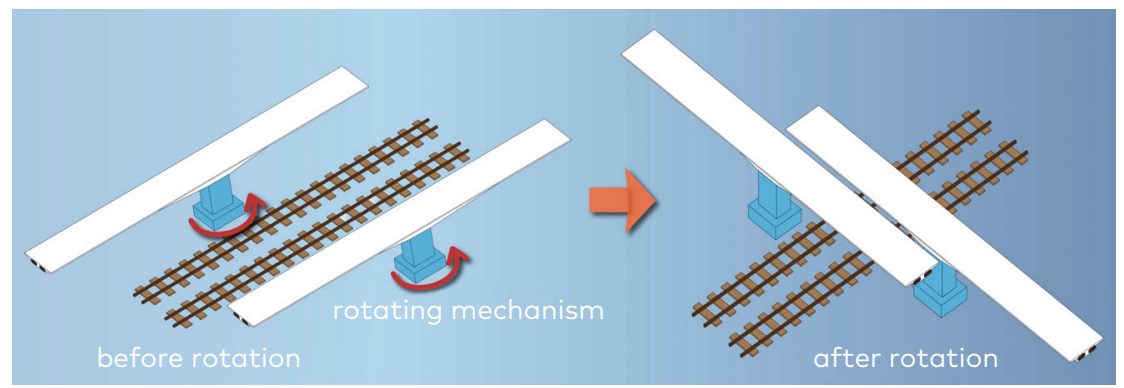

Figure 1. Illustration of the rotation superstructure construction method
An Efficient

Contact Model for Rotating

Mechanism Analysis and Design in Bridge Construction 

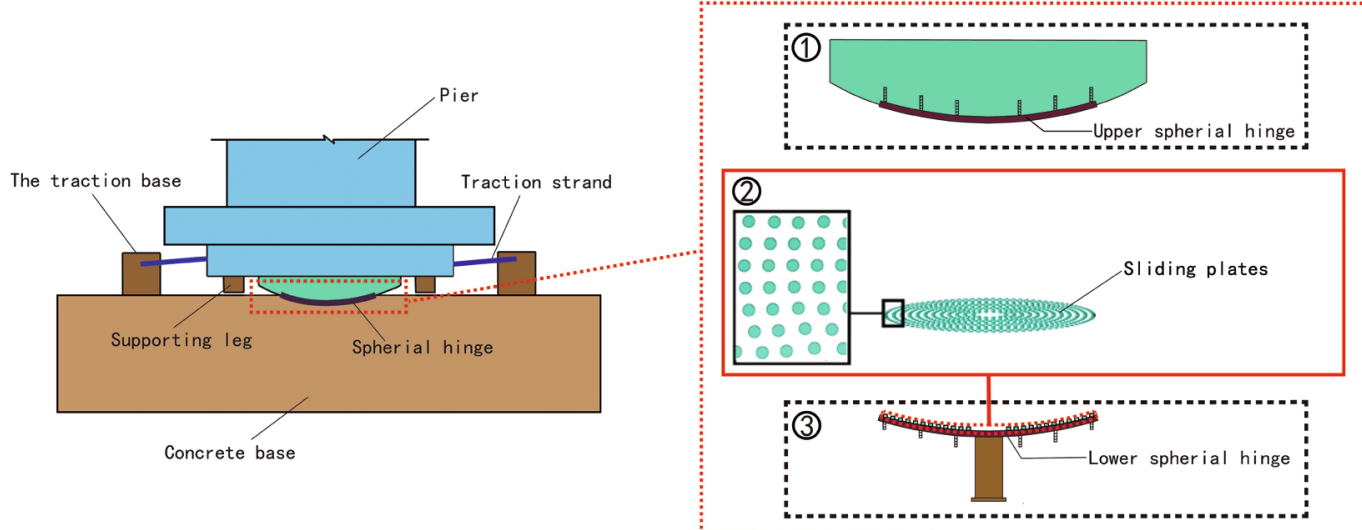

Note: the base is fixed during the simulation.

Figure 2. Configuration of the rotating mechanism

the lower spherical hinge-component. The spherical hinge-component are steel plates embedded within concrete blocks, as shown in Figure 2. Thus, the conforming contacting surfaces are formed by the two spherical steel plates. The spherical hinge provides the following advantages:

1. a large contact area, and

2. stability against overturning.

However, the friction coefficient of the metal is relatively high; thus, a perforated-steel plate with inserted slide plates is introduced between the two components. In this case, the perforated plate is welded to the lower component; then the upper component articulates over the slide plates installed in the perforations, as shown in Figure 3. In this sense, the hinge is expected to mimic biological joints in which soft low-friction articulating surface or insert (as in knee meniscus) intervene at the interface. The slide plates are made of Polytetrafluoroethylene (PTFE) (Sawyer et al., 2003), which is a synthetic fluoropolymer of tetrafluoroethylene. Polytetrafluoroethylene has one of the lowest friction coefficients (with a range of 0.01 to 0.10 ) of any solid with a low-cost expense, thus reducing the overall friction of the mechanism. Therefore, the efficient design for the rotation mechanism is when the pressure in each slide plate is the same, and the stress distribution within each slide-plate is uniform, so the frictional wear of the slide plates is uniform. It notes that the spherical hinge and the slide plates stay in the bridge after the rotation. 


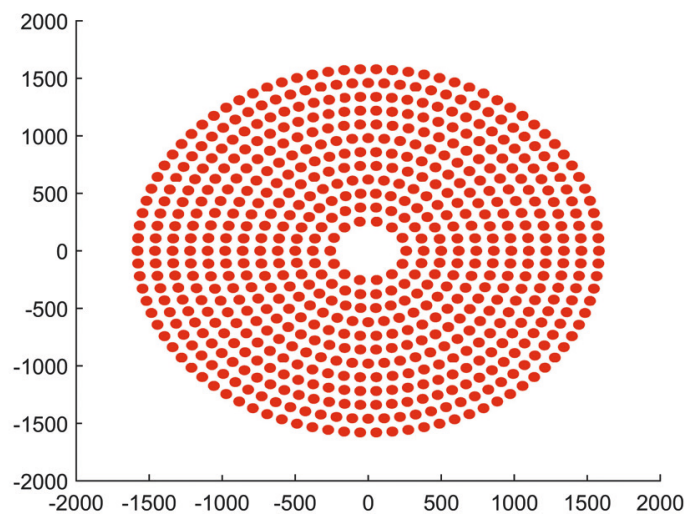

An Efficient

Contact Model for Rotating Mechanism Analysis and Design in Bridge Construction

Note: units in $\mathrm{mm}$.

a) the slide plates distribution in the spherical hinge

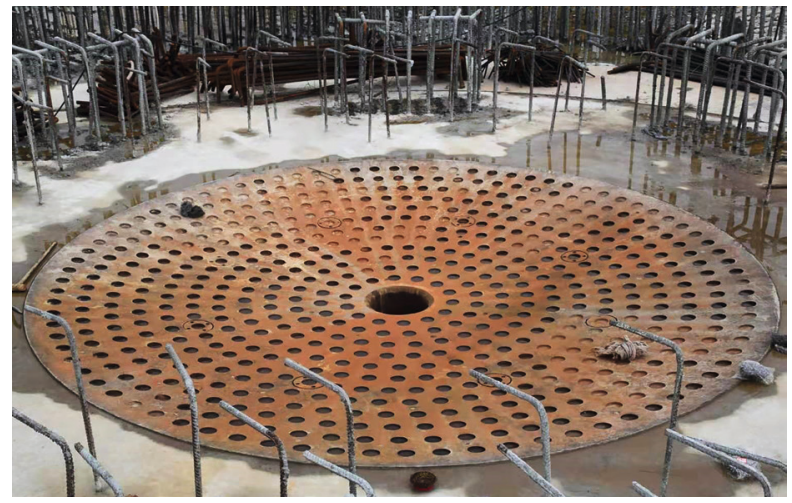

b) spherical hinge with preformed holes for the slide plates

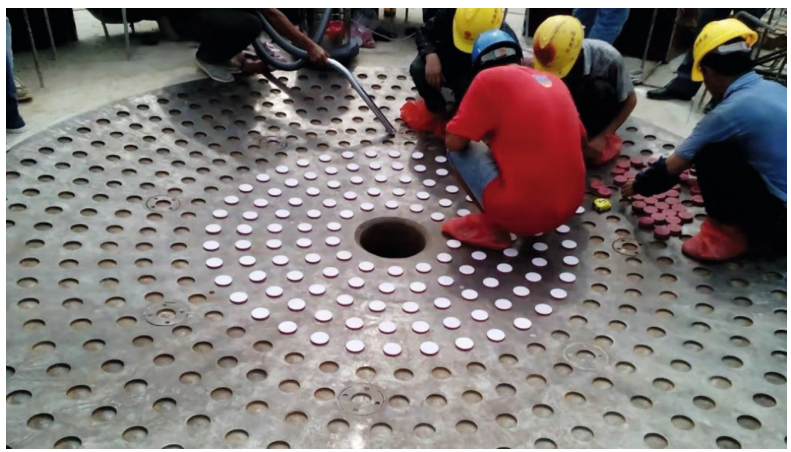

c) installation of the slide plates

Figure 3. Slide plates distribution on the surface 


\subsection{Finite Element Contact Model of the rotational mechanism}

The Finite Element Method has been widely used in broad engineering areas due to its accuracy and efficiency. Thus, the FEM was used to model the contact behaviour of the rotating mechanism and verify the results obtained by the proposed method in the next section. For completeness, the mathematical foundation of the FEM for the contact problem of elastic bodies is introduced briefly (Wang, 2003; Wriggers \& Zavarise, 2004). The contact problem is highly nonlinear. It often experiences three types of nonlinearities, i.e., material nonlinearity, contact nonlinearity, and geometric nonlinearity. Contact boundary constraints are unique to contact problems. The incremental theory is used in FEM where the loading is divided into many increments due to the nonlinearity. In each loading increment $\varnothing t$, the contact boundary constraint (Figure 4) is expressed as:

$$
\begin{gathered}
u^{A}{ }_{N}-u^{B}{ }_{N}+g_{N}=0, \\
u_{T}^{A}-u_{T}^{B}=0,
\end{gathered}
$$

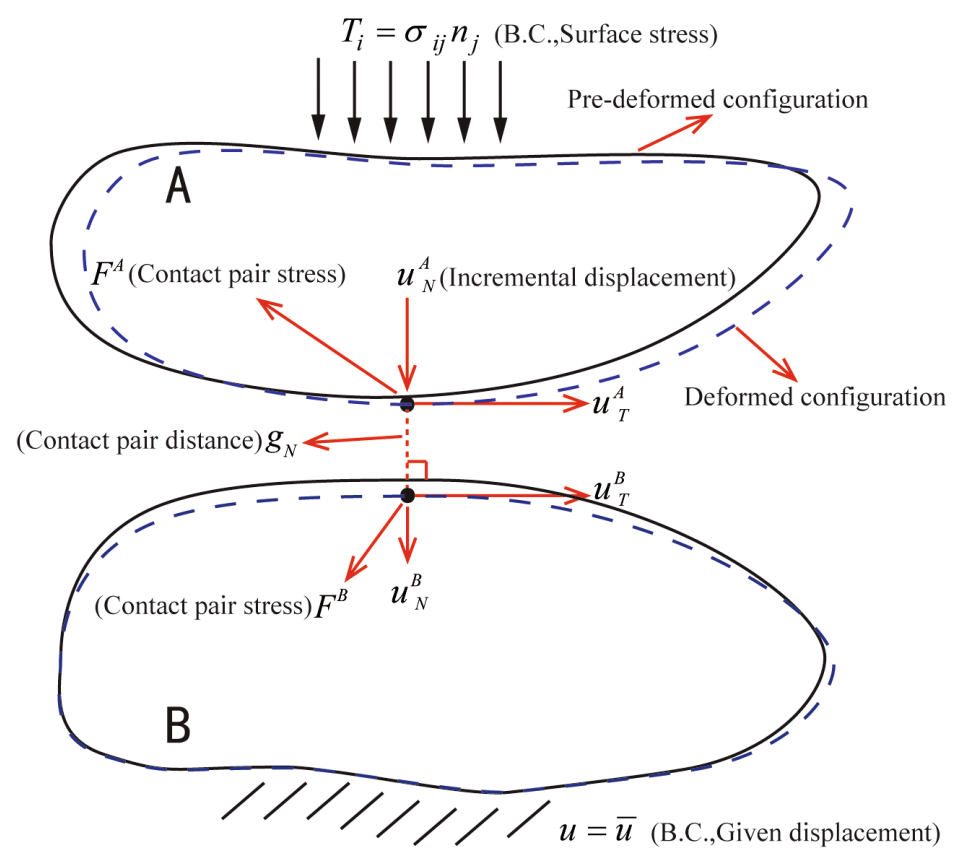

Figure 4. Typical contact problem and boundary conditions 
where $u^{A}{ }_{N}$ and $u^{B}{ }_{N}$ represent the incremental normal displacement vectors of the contact bodies $A$ and $B$ on the contact surface, respectively; $u_{T}^{A}$ and $u_{T}^{B}$ represent the incremental tangential displacement vectors of the contact bodies $A$ and $B$, respectively; $g_{N}$ is the distance vector between the contact points. Accordingly, the constrained partial differential equation is rewritten as the following weak form according to the virtual work principle in Lagrangian description (Wang, 2003):

$$
\begin{aligned}
& \sum_{r=}^{A, B}\left[\int_{t+\Delta t V^{r}}{ }^{t+\Delta t} \tau_{i j}^{r} \delta_{t+\Delta t} e_{i j}^{r t+\Delta t} \mathrm{~d} V\right]+ \\
& \sum_{r=}^{A, B}\left[\int_{{ }^{t+\Delta t} S_{\sigma}{ }^{r}{ }^{t+\Delta t}{ }^{t+\Delta t}} T_{i}^{r} \delta u_{i}^{r}{ }^{t+\Delta t} \mathrm{~d} S\right]+ \\
& \int_{{ }^{t+\Delta t} S_{c}}{ }^{t+\Delta t} F_{J}^{A}\left(\delta u_{J}^{A}-\delta u_{J}^{B}\right)^{t+\Delta t} \mathrm{~d} S=0
\end{aligned}
$$

where $\tau_{i j}$ is the stress tensor and $e_{i j}$ is the strain tensor; $i, j$, and $J$ are tensor/vector index; $T_{i}$ is the surface stress vector for contact solid $A$ and $B ; F_{J}^{A}$ is the contact stress vector at the contacting surface (Figure 4). The upper/lower left index of the tensor/vector $(t+\Delta t)$ is the incremental step expressed in time based on the Lagrangian description. The first term in the Eq. (2) is the virtual strain energy; the second term is the external virtual work produced by the surface force; the third term is the contact force generated on the contact surface virtual work.

The Lagrange multiplier method is introduced to consider additional constraints expressed in Eq. (1), and the corresponding modified functional variation of the displacement increment is as follows:

$$
\begin{aligned}
& \sum_{r=}^{A, B}\left[\int_{t+\Delta t} V^{r}{ }^{t+\Delta t} \tau_{i j}^{r} \delta_{t+\Delta t} e_{i j}^{r}{ }^{t+\Delta t} \mathrm{~d} V\right]+ \\
& \sum_{r=}^{A, B}\left[\int_{\left.{ }^{t+\Delta t} S_{\sigma}^{r}{ }^{r+\Delta t}{ }^{t+\Delta t} T_{i}^{r} \delta u_{i}^{r}{ }^{t+\Delta t} d S\right]+},\right. \\
& \int_{t+\Delta t}{ }^{t+\Delta t} \lambda_{c}^{A}\left(\delta u_{J}^{A}-\delta u_{J}^{B}\right)^{t+\Delta t} \mathrm{~d} S=0
\end{aligned}
$$

where $A_{J}^{A}$ is the Lagrange multiplier. The first term of the Eq. (3) is the virtual strain energy; the second term is the external virtual work produced by the surface force; and the third term is the "virtual work" produced by the constrained variable (i.e., Lagrange multiplier variable). Eqs. (2) and (3) are equivalent, including the relationship between the equivalent node force on the contact interface and the Lagrange multiplier variable. If the entire domain is discretised, and the node displacement is used as the primary variable of the shape function, the final FE solution Eq. (4) is as follows:
An Efficient

Contact Model for Rotating Mechanism Analysis and Design in Bridge Construction 


$$
\left[\begin{array}{cc}
{ }_{0}^{t} K_{L}+{ }_{0}^{t} K_{N L} & K_{c \lambda} \\
K_{c \lambda}^{T} & 0
\end{array}\right]\left(\begin{array}{c}
u \\
{ }^{t+\Delta t} \lambda
\end{array}\right)=\left(\begin{array}{c}
{ }^{t+\Delta t} Q_{L}-{ }_{0}^{t} F \\
-{ }^{t} \bar{g}
\end{array}\right),
$$

where $K_{L}$ is the linear term of the global stiffness matrix of the contact body, and $K_{N L}$ is the corresponding nonlinear term. ${ }^{t+\Delta t} Q_{L}$ is the equivalent node force produced by the surface stress ${ }_{0}^{t} F$ is the unbalanced node force in the linearisation process produced by the first term of Eqs. (2) and (3). The first equilibrium Eq. (4) of the linear system is essentially the discretised form of Eq. (3), and the second equilibrium Eq. (4) of the linear system is the discretised form of constraint Eq. (1).

The discretised Eq. (4) is solved by the finite element algorithm performed by ABAQUS (Abaqus, 2014). The Finite Element Model for the mechanism is shown in Figure 5.

For the case study considered in this paper, the slide plates have been arranged into 12 concentric circular arrangements to have a total of 634 cylindrical slide plates, each of them with a radius of $30 \mathrm{~mm}$ and a height of $18 \mathrm{~mm}$. These slide plates are placed in concentric circles with uniform spacing along a circumference. This spacing is the same for each circle so that the area ascribed to each slide plate is the same. The expectation is that such a placement results in a uniform loading of each slide plate (which is an incorrect assumption for the case of uniform

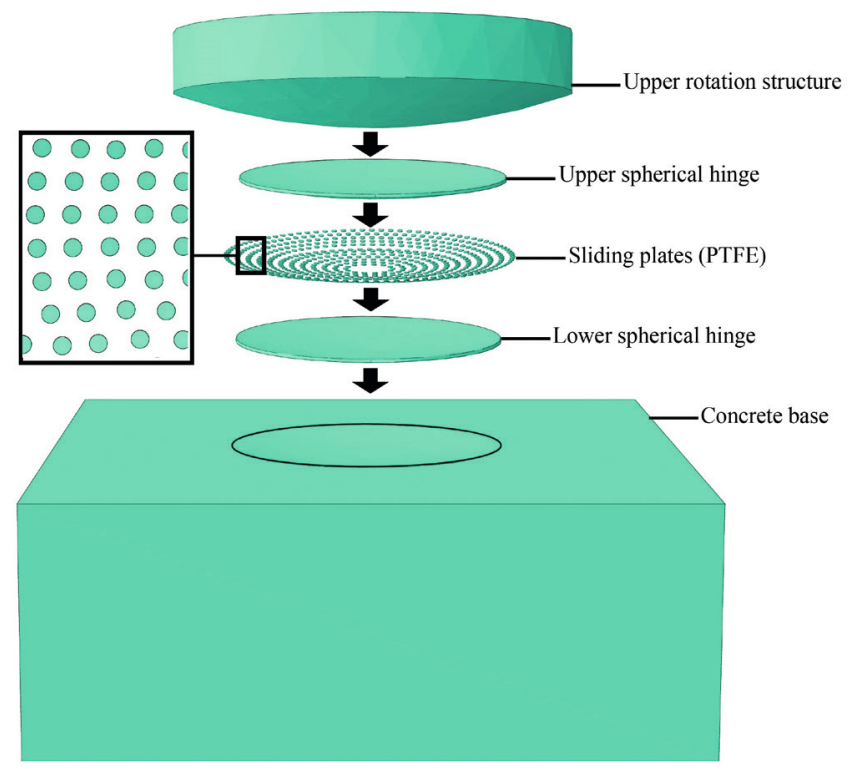

Figure 5. Illustration of the rotating mechanical components in the Finite Element Model 


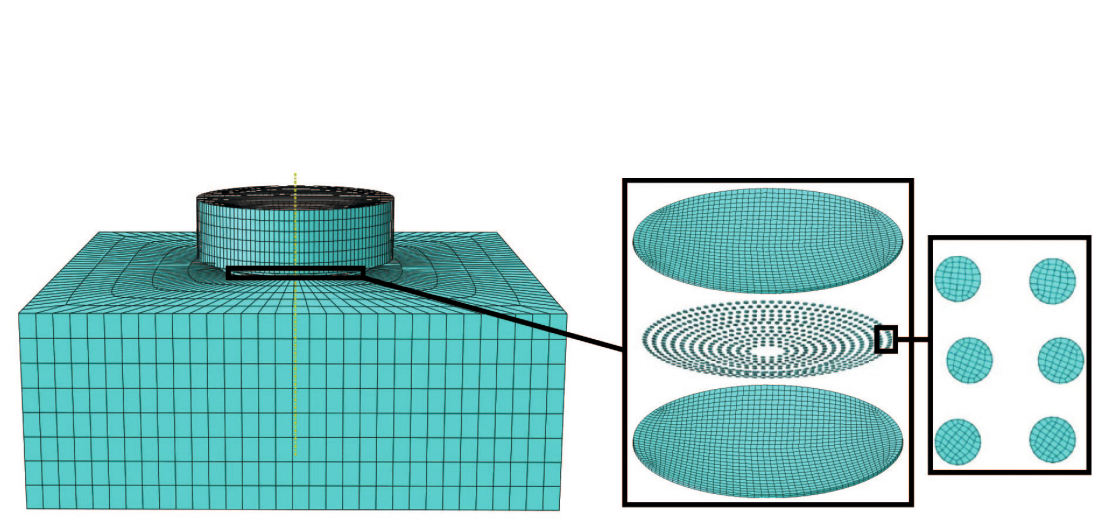

Note: the base is fixed during the simulation.

Figure 6. Finite Element Model of the rotating mechanism

height, as shown later in Section 2). The slide plates are inserted into the preformed holes in the lower spherical hinge, as seen in Figures 3a-3c. This case study is inspired by the Mozhan Bridge, located in Maoming city, China. The bridge includes two side bridges with the same structural details. Two halves of superstructures weigh 10500 tons and are supported by the rotating mechanism. After the construction of the superstructures has been completed, it needs to rotate the mechanism 90 degrees to the desired position.

In the Finite Element Model, the Hexagonal eight-node element (C3D8R) is used to discretise all the parts (Figure 6). During the simulation, the base is fixed. By using trial and error procedure, the mesh size of the element is determined. The total number of elements in the model is 160 596. For each spherical shell, the mesh size is $0.050 \mathrm{~m}$, and the number of elements is 8352 . For each slide plate, the mesh size and the number of elements are $0.006 \mathrm{~m}$ and 186, respectively. The slide plates are tied to the lower spherical hinge and have a "surface to surface" contact with the upper spherical hinge. The material parameters used in this paper is found in Table 1.

Table 1. Material parameters of the Finite Element Model

\begin{tabular}{lcc}
\hline \multirow{2}{*}{ Parts } & \multicolumn{2}{c}{ Parameters of the Finite Element Model } \\
\cline { 2 - 3 } & Elasticity modulus, MPa & Poisson ratio \\
\hline Upper rotation structure & $3.25 \cdot 10^{4}$ & 0.2 \\
\hline Upper spherical hinge & $2.10 \cdot 10^{5}$ & 0.3 \\
\hline Lower spherical hinge & $2.10 \cdot 10^{5}$ & 0.3 \\
\hline Sliding plates (PTFE) & 1400 & 0.4 \\
\hline Concrete base & $3.25 \cdot 10^{4}$ & 0.2 \\
\hline
\end{tabular}




\subsection{The efficient contact models}

The above FEM is complex from the design viewpoint since it needs many degrees of freedom and requires the solution of a nonlinear contact problem, which is computationally expensive. The hinge design is optimised by using the iterative method or trial and error method. Using so large FEMs is far beyond the typical design practice where an ad hoc approach is often used. From the viewpoint of practice, therefore, simplified yet robust approaches are desired.

A way forward is by adopting the classic contact mechanics (Johnson, 1985). In this simplification, the surface (or interfacial) geometry of the contact of two elastic solids is treated as a rigid solid with an elastic solid by introducing the composite surface $H$, which is expressed as:

$$
H(x, y)=H_{1}(x, y)+H_{2}(x, y),
$$

where $H_{1}$ and $H_{2}$ are the surface geometry in a Cartesian coordinate system. For the case study considered in this paper, the contact occurs between the upper spherical hinge component (steel) and the PTFE slide plates embedded in the lower spherical hinge component. Thus, $H_{1}$ is the outer surface profile of the upper spherical hinge component, and $\mathrm{H}_{2}$ is the inner surface profile of the lower spherical hinge component with slide plates (Figure 7a). $H$ is the composite surface profile, which is the sum of $H_{1}$ and $H_{2}$. It notes that $H_{1}=R^{\prime}=7992 \mathrm{~mm}$ and $H_{2}=$ $R-S_{h}=7992 \mathrm{~mm}$, where $S_{h}=8 \mathrm{~mm}$ is the raised part of the embedded slide plate; thus, the surface profile $H$ is a flat surface. The equivalent

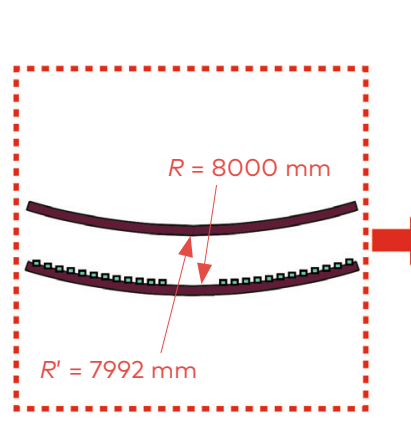

a) the original contact model profile

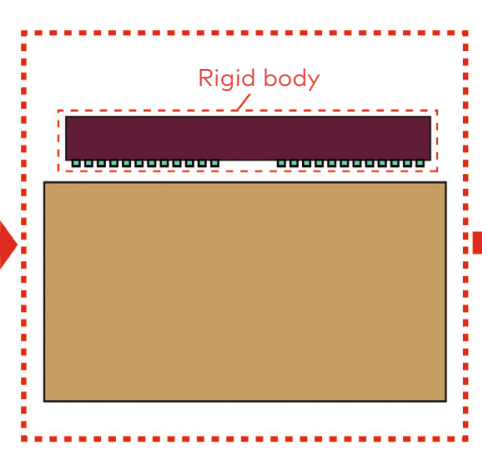

b) transformed into contact between a rigid and an elastic block

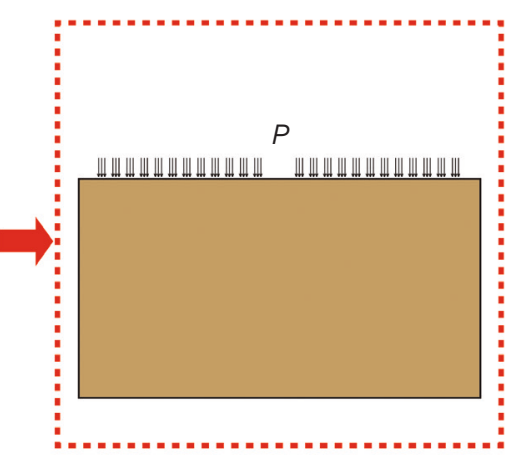

c) further transformed into an elastic problem of half-space

Figure 7. Simplification of the contact model: from multi-bodies contact problem to classic elasticity 
elasticity modulus $E^{*}$ and Poisson ratio $v$ is given by Eq. (6) (Ciavarella et al., 2019; Johnson, 1985; Persson, 2007):

$$
\frac{1-v^{2}}{E^{*}}=\frac{1-v_{1}^{2}}{E_{1}}+\frac{1-v_{2}^{2}}{E_{2}},
$$

where $E_{1}$ and $E_{2}$ are the elastic moduli for the contact body 1 and 2, respectively; $v_{1}$ and $v_{2}$ are the Poison ratios for the contact body 1 and 2 , respectively.

This simplification is implemented here for the analysis. In Figure 7, the contact of slide plates with the upper hinge component is idealised as the contact of rigid punch with rounded end indenting a flat elastic half-space as stated in Eqs. (5)-(6). In this idealisation, the contact of a desired rounded rigid punch with flat elastic half-space results in uniform pressure distribution. This idealisation is especially appropriate since the elastic modulus of slide plates is two orders of magnitude smaller than the upper hinge component made of steel. Thus, the significant contribution to the deformation is from the slide plates only. Therefore, the contact problem is transformed to of determining the displacement profile of elastic half-space subjected to uniform pressure on a set of circular areas.

In this case, the normal surface displacement produced by a normal concentrated force $P$ is expressed as:

$$
\bar{u}_{z}=\frac{1-v^{2}}{\pi E} \frac{P}{r},
$$

where $r$ is the distance from the origin.

Since the contact force produced by the slide plate is within the circular area, thus, the total surface displacement due to pressure $p(s, \varphi)$ is obtained as follows:

$$
\bar{u}_{z}=\frac{1-v^{2}}{\pi E} \iint p(s, \varphi) \mathrm{d} s \mathrm{~d} \varphi,
$$

where $(s, \varphi)$ are polar coordinates.

For the uniform circular contact pressure with radius $r, P$ is a constant. Eq. (8) yields the following results:

$$
\bar{u}_{z}=\frac{4\left(1-v^{2}\right) p a}{\pi E} E\left(\frac{r}{a}\right),(r<a),
$$

where $E_{e}\left(\frac{r}{a}\right)$ is the complete elliptic integral of the second kind with the modulus $\left(\frac{r}{a}\right)$, and $E_{e}$ is expressed as:
An Efficient

Contact Model for Rotating Mechanism Analysis and Design in Bridge Construction 


$$
E_{e}\left(\frac{r}{a}, \varphi\right)=\int_{0}^{\frac{\pi}{2}} \sqrt{1-\left(\frac{r}{a}\right)^{2} \sin ^{2} \varphi \mathrm{d} \varphi}
$$

At the edge of the circle, the displacement $u_{z}$ has a simple expression:

$$
\bar{u}_{z}=\frac{4\left(1-v^{2}\right) p a}{\pi E},(r=a) \text {. }
$$

Outside the pressure area, the displacement $u_{z}$ has the following expression as:

$$
\bar{u}_{z}=\frac{4\left(1-v^{2}\right) p r}{\pi E}\left\{E_{e}\left(\frac{a}{r}\right)-\left(1-\frac{a^{2}}{r^{2}}\right) K_{e}\left(\frac{a}{r}\right)\right\},(r>a),
$$

where $K_{e}\left(\frac{a}{r}\right)$ is the complete elliptic integral of the first kind with the modulus $\left(\frac{a}{r}\right)$, and $K_{e}$ is expressed as:

$$
K_{e}\left(\frac{r}{a}, \varphi\right)=\int_{0}^{\frac{\pi}{2}} \frac{1}{\sqrt{1-\left(\frac{r}{a}\right)^{2} \sin ^{2} \varphi}} \mathrm{d} \varphi
$$

As shown in Figure 8, the displacement induced by the uniform pressure decreases with $r$, and the indentation below a slide plate is affected by its neighbour slide plates.

Since the closed-form solution for the complete elliptical integral is absent and the numerical integral results inexpensive computation,

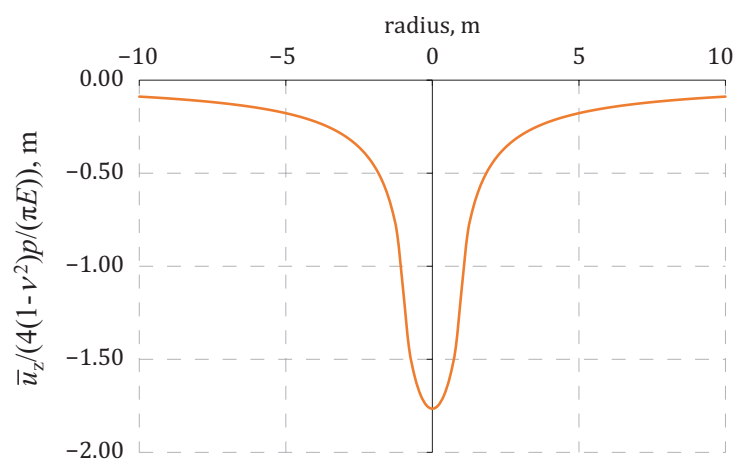

Note: pressure area $a=1 \mathrm{~m}$, and Poisson ratio $v=0.35$.

Figure 8. Demonstration of the displacement induced by the uniform pressure 
the authors have used the approximation (Cody, 1965; Hastings Jr et al., 1955), where $K_{e}$ is expressed as:

$$
K_{e}(k)=\sum_{i=0}^{n} a_{i} \eta^{i}+\ln \left(\frac{1}{\eta}\right) \sum_{j=0}^{m} b_{j} \eta^{j}
$$

An Efficient

Contact Model for Rotating Mechanism Analysis and Design in Bridge Construction

where $a_{0}=\ln 4, \sum_{i=0}^{n} a_{i}=\frac{\pi}{2}$, and $b_{0}=\frac{1}{2}$ and $m, n$ are approximation numbers. A larger number leads to a smaller error. Here, $m$ is considered equal to $n . E_{e}$ is expressed as the following form:

$$
E_{e}(k)=\sum_{i=0}^{n} c_{i} \eta^{i}+\ln \left(\frac{1}{\eta}\right) \sum_{j=0}^{m} d_{j} \eta^{j},
$$

where $c_{0}=1, \sum_{i=0}^{n} c_{i}=\frac{\pi}{2}$, and $d_{0}=0$ and $\eta$ is the square of the complementary modulus, which is expressed as:

$$
\eta=1-k^{2} \text {. }
$$

Eqs. (7)-(15) give an efficient method to get the deformation corresponding to uniform pressure due to a single slide plate. The authors then superpose the displacement by all the slide plates to get the final displacement field. As a result, the corresponding shape of the slide plate is obtained.

\subsection{Optimal design procedure}

The method, as mentioned above, is immediately applied to the engineering design for the rotational mechanism. Here, the optimal objective is to obtain the height distribution of the slide plates for a given arrangement (Figure 3a). A summary of the optimal design procedure for use in practice is given in the flowchart in Figure 9. For a given superstructure and pre-specified slide plate diameter (typically these are available in standard sizes), the first step is to determine the slide plate position to ensure uniform ascribed area, as noted in Section 1.2. The method described in the previous section is then applied to determine the height distribution of the slide plates. Typically, for each slide plate, Eqs. (9), (11) and (12) are evaluated numerically. The overall displacement field of the elastic block is then determined by the superposition of the slide plate fields. It is clear that due to the superposition, the indentations produced below each slide plate is affected by its neighbours. The resulting indentation depth is the correct height profile for the slide plates, which gives the assumed 

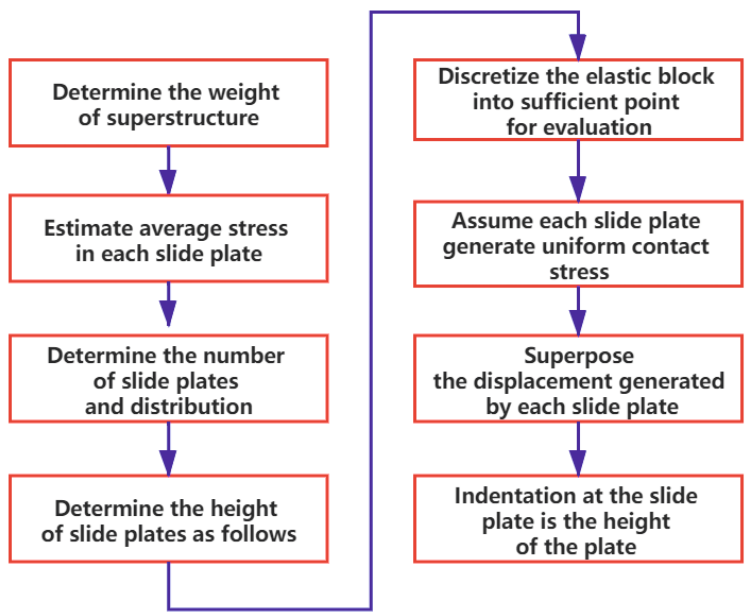

Figure 9. Flowchart of the optimal design based on the proposed contact method

uniform pressure distribution. It is particularly true since the slide plates significantly contribute to the overall deformation as they are two orders of magnitude softer.

\section{Results and discussions}

\subsection{The input information for the case study of the rotation mechanism}

In the following result, the case study for Maozhan Bridge is discussed. The input parameters for the FEM is found in Table 1. The boundary condition for the FEM is shown in Figure 5; where the base is fixed. The surface loading stress (11.34 MPa) is uniformly distributed on the top surface. The efficient contact model parameters are also based on the Maozhan Bridge, where the equivalent elastic modulus $E=1450 \mathrm{MPa}$ and Poisson ratio $v=0.35$. Moreover, the compressive yield stress of the plates is $100 \mathrm{MPa}$.

\subsection{Slide plates with uniform heights}

When the slide plates have a uniform height of $18 \mathrm{~mm}$, the force of each slide plate is different, where the average stress of the slide plate 


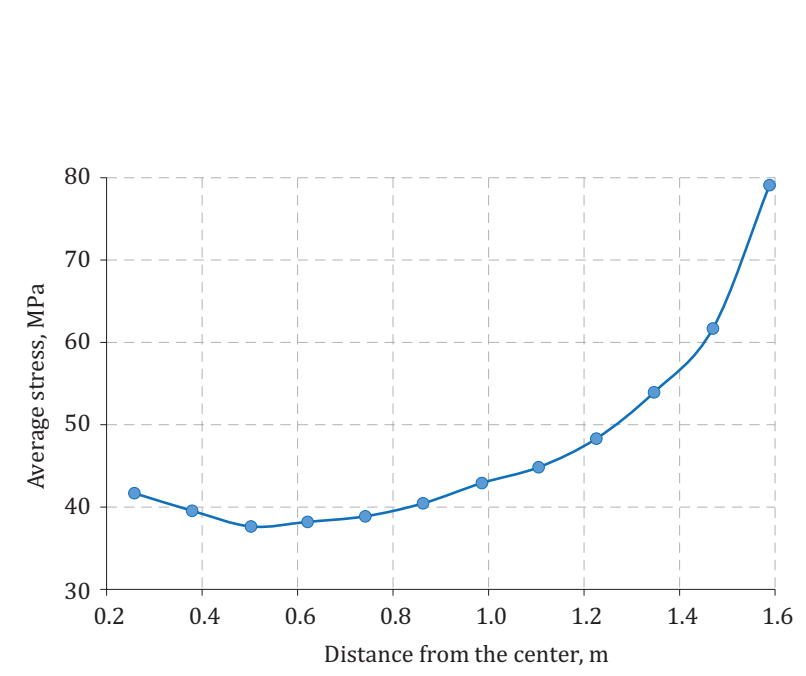

Shiping Huang,

An Efficient

Contact Model

for Rotating

Mechanism Analysis and Design in Bridge Construction

Figure 10. Stress distribution of each slide plate for the original design

(force divided by the area) is shown as in Figure 10. Furthermore, as shown in Figure 11, the stress distribution is ununiform within each slide plate. It is observed that the maximum stress is about $113 \mathrm{MPa}$, while the average stress is about $57.40 \mathrm{MPa}$. The stress concentration may result in local damage. Even though some researchers believe that friction coefficient is independent of the normal stress (Huang \& Misra, 2013; Urbakh et al., 2004), high contact stress under a given overall load may lead to local damage, which in return results in asperity interlocking effect (Huang \& Misra, 2013) or ploughing effect (Johnson, 1985). Therefore, the desired normal stress distribution follows a uniform stress distribution, and the authors use the proposed method to get it.
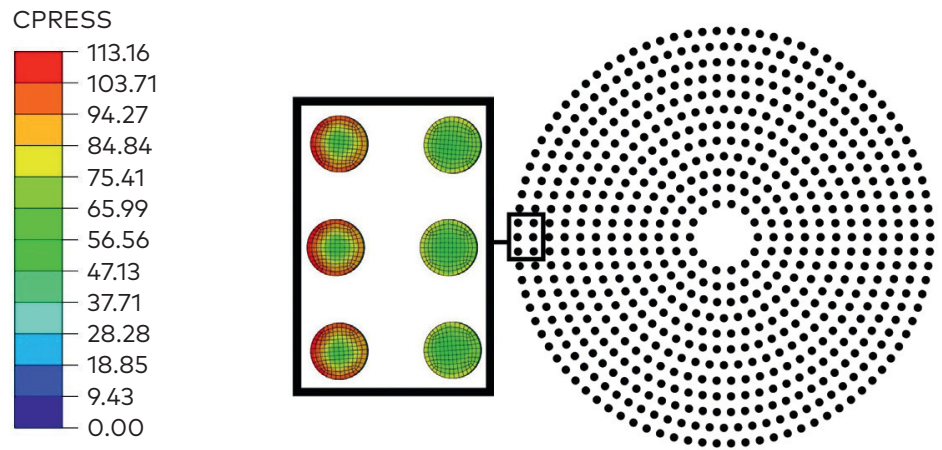

Figure 11. Stress distribution of the rotating mechanism with slide plates 


\subsection{Optimal heights of the slide plates using the proposed contact method}

To avoid stress concentration, the authors used the proposed method to get the desired height distribution of the slide plates. As discussed before, the indentations of the elastic block are the corresponding heights of the slide plates. Displacement, which is the expecting optimal profile, can be obtained quickly under the uniform pressure. Based on the discussion in Section 1.4, the displacement fields induced by a set of uniform pressures are obtained. And then, the corresponding displacement matches the indentations of the slide plates. Consequently, the heights of the slide plates are determined.

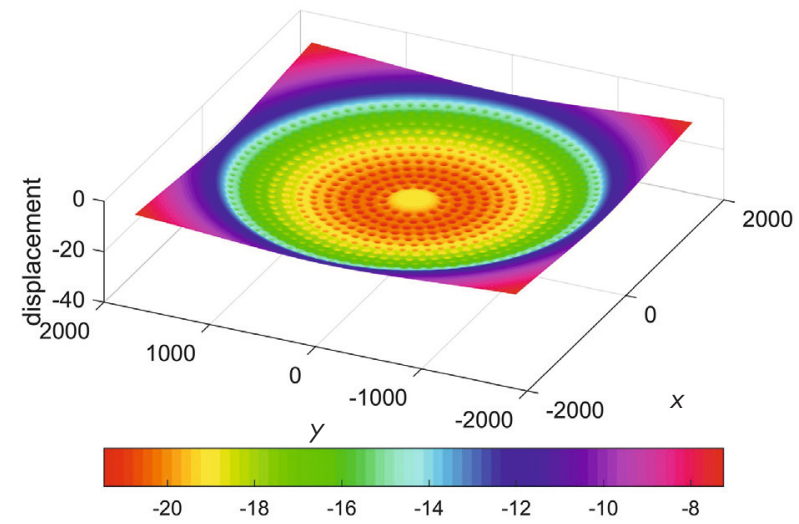

Note: units in $\mathrm{mm}$.

Figure 12. The shape of the slide plate using the half-space theory

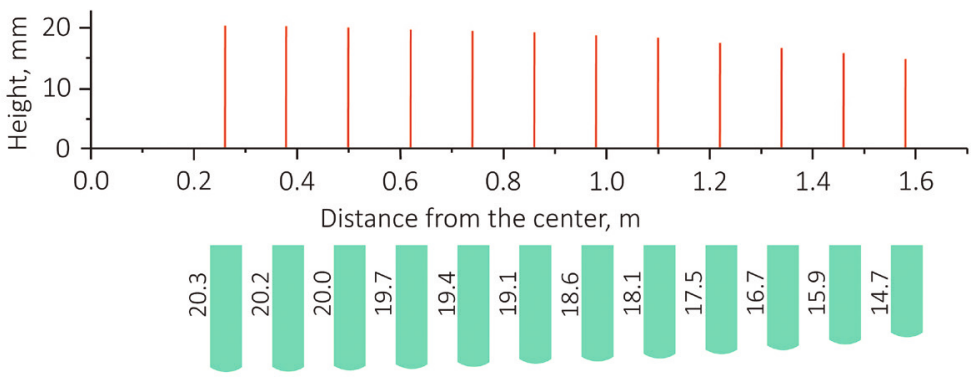

Note: from the centre to the edge.

Figure 13. Height distribution for the profile of slide plates 


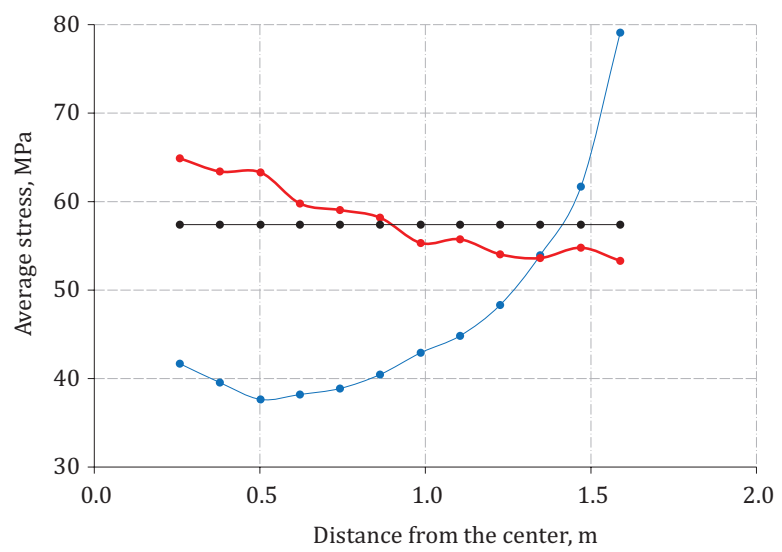

An Efficient

Contact Model for Rotating Mechanism Analysis and Design in Bridge Construction

$\rightarrow$ before optimise $\rightarrow$ after optimise $\rightarrow$ the mean value after optimise

Figure 14. Stress distribution at each slide plate for the original model and the optimal model based on finite element analysis

Notably, the deformation caused by uniform pressure induced by 634 slide plates can be seen in Figure 12 (assume each slide plate yields the same uniform pressure). Then according to this indention (deformation), the height of the slide plate can be back-calculated. Figure 13 shows the profile (height distribution) of the slide plate, giving the uniform contact stress when contacting the surface.

For evaluating the proposed approach, the slide plate in Figure 13 is used as the input for the FEM. In Figure 14, the authors found that the stress at each slide plate tends to be uniform. Moreover, the stress distribution is more uniform (Figure 15), where the maximum stress
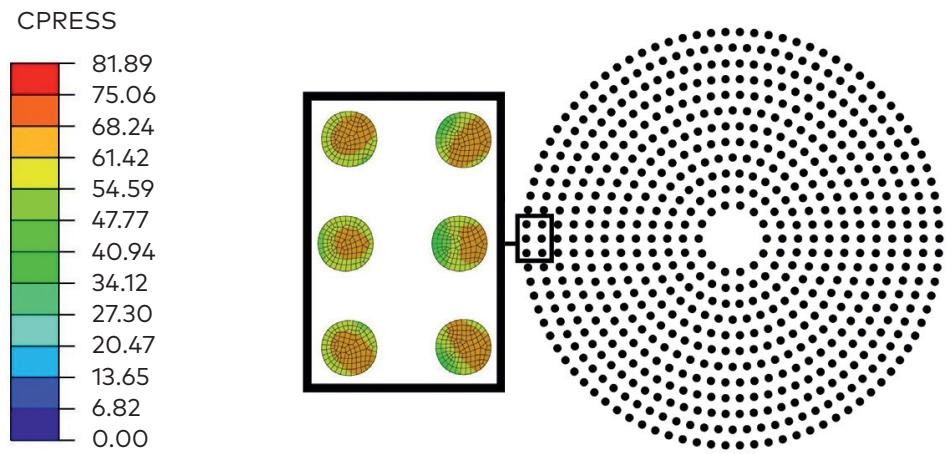

Figure 15. Stress distribution of the rotating mechanism with slide plates after the optimal process 
is about $80 \mathrm{MPa}$, which is two-thirds of the maximum stress using traditional design using uniform heights. It indicates that the optimal design is advantageous.

\section{Conclusions}

This paper has analysed a massive contact problem critical to the rotation superstructure construction method in bridge engineering. It is shown that an understanding of the contact behaviour is important for the surface design in the rotating mechanism. The conclusion is drawn as follows:

1. The proposed contact model reduces the geometry complexity, which transforms the multi-bodies contact problem into a classic elasticity problem. Based on the desired stress distribution, the superposition approach is used to obtain the contact surface to prevent the iterative method, which involves the expensive computational expense.

2. The finite element analysis results show that the contact surface generated by the proposed contact model meets the design requirement, i.e., a uniform contact pressure distribution is given under the slide plates.

3. The revised design based on the proposed method reduces the material cost of the slide plates and reduces friction and avoids the local damage.

Therefore, the proposed method can be used in a broad area involving contacting surface design, especially in the rotating mechanism design for bridge construction.

\section{Funding}

This work was supported by the National Natural Science Foundation of China under Grant No. 11672108 and 11911530692.

\section{REFERENCES}

Abaqus, V. (2014). 6.14 Documentation. Dassault Systemes Simulia Corporation, 651, 6-2.

Banerjee, P. K., \& Butterfield, R. (1981). Boundary element methods in engineering science 17, p. 578. London: McGraw-Hill. 
Barber, J. R., \& Ciavarella, M. (2000). Contact mechanics. International An Efficient Journal of Solids and Structures, 37(1-2), 29-43. https://doi.org/10.1016/ S0020-7683(99)00075-X

Berman, D., Deshmukh, S. A., Sankaranarayanan, S. K., Erdemir, A., \& Sumant, A. V. (2015). Macroscale superlubricity enabled by graphene nanoscroll formation. Science, 348(6239), 1118-1122. https://doi.org/10.1126/science.1262024

Brebbia, C. A., \& Walker, S. (2016). Boundary element techniques in engineering. Elsevier.

Bush, A. W., Gibson, R. D., \& Thomas, T. R. (1975). The elastic contact of a rough surface. Wear, 35(1), 87-111. https://doi.org/10.1016/0043-1648(75)90145-3

Ciavarella, M., Joe, J., Papangelo, A., \& Barber, J. R. (2019). The role of adhesion in contact mechanics. Journal of the Royal Society Interface, 16(151), 20180738. https://doi.org/10.1098/rsif.2018.0738

Cody, W. J. (1965). Chebyshev approximations for the complete elliptic integrals $K$ and E. Mathematics of Computation, 19(89), 105-112. https://doi.org/10.1090/S0025-5718-1965-0171370-4

Greenwood, J. A., \& Williamson, J. P. (1966). Contact of nominally flat surfaces. Proceedings of London. Series A. Mathematical and Physical Sciences, 295(1442), 300-319. https://doi.org/10.1098/rspa.1966.0242

Hastings Jr, C., Wayward, J. T., \& Wong Jr, J. P. (1955). Approximations for digital computers. Princeton University Press.

Hertz, H. (1881). On the contact of elastic solids I. Reine Angew. Mathematik, 92, 156-171.

Hertz, H. (1896). “On hardness," Verh. Ver. Beförderung Gewerbe Fleisses 61, 1882, p. 410. Translated and reprinted in English in Hertz's Miscellaneous Papers, Macmillan \& Co, London, 1896, Ch. 6.

Huang, S. (2017). Evolution of the Contact Area with Normal Load for Rough Surfaces: from Atomic to Macroscopic Scales. Nanoscale Research Letters, 12(1), 1-8. https://doi.org/10.1186/s11671-017-2362-8

Huang, S., \& Misra, A. (2013). Micro-macro-shear-displacement behavior of contacting rough solids. Tribology Letters, 51(3), 431-436. https://doi.org/10.1007/s11249-013-0178-y

Hyun, S., Pei, L., Molinari, J. F., \& Robbins, M. O. (2004). Finite-element analysis of contact between elastic self-affine surfaces. Physical Review E, 70(2), 026117. https://doi.org/10.1103/PhysRevE.70.026117

Johnson, K. L. (1985). Contact mechanics. Cambridge university press. Longuet-Higgins, M. S. (1957a). Statistical properties of an isotropic random surface. Philosophical Transactions of the Royal Society of London. Series A, Mathematical and Physical Sciences, 250(975), 157-174. https://doi.org/10.1098/rsta.1957.0018

Longuet-Higgins, M. S. (1957b). The statistical analysis of a random, moving surface. Philosophical Transactions of the Royal Society of London. Series A, Mathematical and Physical Sciences, 249(966), 321-387.

https://doi.org/10.1098/rsta.1957.0002 
Luan, B., \& Robbins, M. O. (2005). The breakdown of continuum models for mechanical contacts. Nature, 435(7044), 929-932. https://doi.org/10.1038/nature03700

Mindlin, R. D. (1953). Elastic spheres in contact under varying oblique forces. Journal of Applied Mechanics, 20(3), 327-344. (in Japanese)

Misra, A., \& Huang, S. (2011). Effect of loading induced anisotropy on the shear behavior of rough interfaces. Tribology International, 44(5), 627-634. https://doi.org/10.1016/j.triboint.2010.12.010

Mo, Y., Turner, K. T., \& Szlufarska, I. (2009). Friction laws at the nanoscale. Nature, 457(7233), 1116-1119. https://doi.org/10.1038/nature07748

Nayak, P. R. (1971). Random Process Model of Rough Surfaces. Journal of Lubrication Technology 93(3), 398-407. https://doi.org/10.1115/1.3451608

Nayak, P. R. (1973a). Random process model of rough surfaces in plastic contact. Wear, 26(3), 305-333. https://doi.org/10.1016/0043-1648(73)90185-3

Nayak, P. R. (1973b). Some aspects of surface roughness measurement. Wear, 26(2), 165-174. https://doi.org/10.1016/0043-1648(73)90132-4

Pastewka, L., Sharp, T. A., \& Robbins, M. O. (2012). Seamless elastic boundaries for atomistic calculations. Physical Review B, 86(7), 075459. https://doi.org/10.1103/PhysRevB.86.075459

Persson, B. N. J. (2007). Relation between interfacial separation and load: a general theory of contact mechanics. Physical Review Letters, 99(12), 125502. https://doi.org/10.1103/PhysRevLett.99.125502

Sawyer, W. G., Freudenberg, K. D., Bhimaraj, P., \& Schadler, L. S. (2003). A study on the friction and wear behavior of PTFE filled with alumina nanoparticles. Wear, 254(5-6), 573-580. https://doi.org/10.1016/S0043-1648(03)00252-7

Urbakh, M., Klafter, J., Gourdon, D., \& Israelachvili, J. (2004). The nonlinear nature of friction. Nature, 430(6999), 525-528. https://doi.org/10.1038/nature02750

Wang, X. (2003). Finite element method. Qing Hua University Publishing Company, Beijing. (in Chinese)Wriggers, P., \& Zavarise, G. (2004). Computational contact mechanics. Encyclopedia of Computational Mechanics. https://doi.org/10.1002/0470091355.ecm033

Zhang, J., \& El-Diraby, T. E. (2006). Constructability analysis of the bridge superstructure rotation construction method in China. Journal of Construction Engineering and Management, 132(4), 353-362.

https://doi.org/10.1061/(ASCE)0733-9364(2006)132:4(353) 